$10-11-2019$

\title{
Estimation of Gait Kinematics and Kinetics from Inertial Sensor Data Using Optimal Control of Musculoskeletal Models
}

\author{
Eva Dorschky \\ Friedrich-Alexander University of Erlangen-Nürnberg, eva.dorschky@fau.de \\ Marlies Nitschke \\ Friedrich-Alexander University of Erlangen-Nürnberg \\ Ann-Kristin Seifer \\ Friedrich-Alexander University of Erlangen-Nürnberg \\ Antonie J. van den Bogert \\ Cleveland State University, a.vandenbogert@csuohio.edu \\ Bjoern M. Eskofier \\ Friedrich-Alexander University of Erlangen-Nürnberg
}

Follow this and additional works at: https://engagedscholarship.csuohio.edu/enme_facpub

Part of the Biomechanical Engineering Commons

How does access to this work benefit you? Let us know!

\section{Recommended Citation}

Dorschky, Eva; Nitschke, Marlies; Seifer, Ann-Kristin; van den Bogert, Antonie J.; and Eskofier, Bjoern M., "Estimation of Gait Kinematics and Kinetics from Inertial Sensor Data Using Optimal Control of Musculoskeletal Models" (2019). Mechanical Engineering Faculty Publications. 376.

https://engagedscholarship.csuohio.edu/enme_facpub/376

This Article is brought to you for free and open access by the Mechanical Engineering Department at EngagedScholarship@CSU. It has been accepted for inclusion in Mechanical Engineering Faculty Publications by an authorized administrator of EngagedScholarship@CSU. For more information, please contact library.es@csuohio.edu. 


\title{
Estimation of gait kinematics and kinetics from inertial sensor data using optimal control of musculoskeletal models
}

\author{
Eva Dorschky, Marlies Nitschke, Ann-Kristin Seifer, Antonie J. van den Bogert, \\ Bjoern M. Eskofier
}

\section{A R T I C L E I N F O}

Article history:

Accepted 18 July 2019

Keywords:

Gait analysis

Inertial sensors

Motion capturing

Optimal control

\section{Introduction}

Human gait gives insight into human health and motor control. Gait analysis is commonly used to support medical diagnosis, monitoring the rehabilitation process of patients and quantifying the performance of athletes. In a gold-standard gait laboratory, camera-based systems and force plates are installed to compute gait kinematics and kinetics. These systems are expensive and restricted to a limited field of view in time and space. It is often desired to carry out a high quality analysis under non-laboratory conditions, e.g. for the ambulant assessment of patients or for analyzing athletes' performance on the field.

Body sensor networks with integrated inertial measurement units (IMUs) such as Xsens MVN (Xsens Technologies BV, Enschede, NL) promise a quantitative movement analysis in unconstrained environments. However, IMUs suffer from measurement noise and drifting biases making an integration-based analysis difficult (Sabatini et al., 2015). Moreover, inertial sensors provide only kinematic data. Other sensor modalities, e.g. pressure insoles or electromyography (EMG), can be used to estimate additional parameters like joint loads or muscle activity patterns. However, the usage of a large number of sensors increases cost and inhibits natural movements. Overall, two problems need to be addressed: (1) sensor noise and drift and (2) a comprehensive analysis based on a limited number of sensors and sensor modalities.

Filtering- and global optimization-based approaches have been proposed to cope with sensor noise and drift. For example, Roetenberg et al. (2005) presented an extended Kalman filter using sensor fusion. Using kinematic body models and external contacts, errors in the predicted segment positions and orientations could be corrected (Roetenberg et al., 2009). Besides filtering, optimizationbased techniques were proposed to estimate relative positions and orientations of body segments. Koning et al. (2013) solved a global optimization problem to simulate a biomechanical model. During optimization, the difference between orientations of the model segments and orientations computed by sensor fusion of IMU data was minimized. Hence, errors in orientation estimation were directly incorporated into the optimization. Kok et al. (2014) 
estimated the relative position and orientation of each body segment by solving an maximum-a posteriori optimization problem. They modeled accelerometer noise and gyroscope bias using a Gaussian distribution and included biomechanical constraints to obtain a drift-free estimation of joint angles.

All the above described methods provide only gait kinematics. Kinetic analysis is also of interest, which relates to the second above-mentioned challenge. On the one hand, additional sensors like instrumented force shoes (Liedtke et al., 2007; Liu et al., 2010; Schepers et al., 2009) could be used. On the other hand, methods have been proposed to estimate gait kinetics based only on IMU data (reviewed by Ancillao et al. (2018)). Machine learning approaches show promising results for estimating specific gait parameters (Hannink et al., 2016; Wouda et al., 2018). However, the collection of representative training data is time-consuming and sometimes infeasible. Unlike data-driven approaches, musculoskeletal simulation yields interpretable results providing insights into muscular control and mechanical characteristics of movement. To date, Karatsidis et al. (2018) presented the most extensive kinematic and kinetic gait analysis based on IMUs and musculoskeletal modeling. They obtained segment positions and orientations from the Xsens system with 17 IMUs and computed kinematics by constrained optimization. Afterwards, they solved a static optimization problem to obtain the muscle reaction forces for the given computed motion trajectory by minimizing muscle activity subject to dynamic equilibrium constraints. Joint moments and ground reaction forces (GRFs) were estimated using inverse dynamics. Karatsidis et al. (2018) evaluated their method on walking at different speeds. It would be of interest to know how their method performs on highly dynamic movements like running, as they derived muscle forces based on static optimization. However, static optimization cannot account for time-dependent interactions like muscle activation-deactivation dynamics and elastic energy storage in the tendons (Prilutsky and Zatsiorsky, 2002). Furthermore, different tools were sequentially used including the Xsens and Anybody system which limits the applicability of the procedure to these commercial systems.

In contrast, we propose a dynamic optimization which yields a dynamically consistent simulation. Moreover, we would like to reduce the number of necessary tools, processing steps and IMUs to make the analysis practical for clinical and use in sports.

Our goal is to estimate gait kinematics and kinetics directly from raw inertial sensor data performing one global dynamic optimization. Therefore, we formulated an optimal control problem to find movement and muscular control trajectories of gait cycles. The method is based on van den Bogert et al. (2011) and was already applied for estimating knee joint loads from noisy video data (Heinrich et al., 2014). Our contribution is the extension and evaluation of the optimal control simulation to estimate sagittal kinematics and kinetics of the lower limbs from IMU data. First, we describe the planar musculoskeletal model in Section 2.1 and virtual sensor model in Section 2.2. In Section 2.3, the optimal control problem is formulated. We conducted an evaluation study using seven IMUs placed on the lower limbs and an optical motion capturing system including one force plate as the gold standard for purposes of comparison. In total, we recorded ten healthy male subjects walking and running overground at three different speeds each (see Section 2.4).

\section{Methods}

\subsection{Musculoskeletal model and dynamics}

The planar musculoskeletal model consisted of seven rigid segments, one segment representing the head, arms and torso denoted

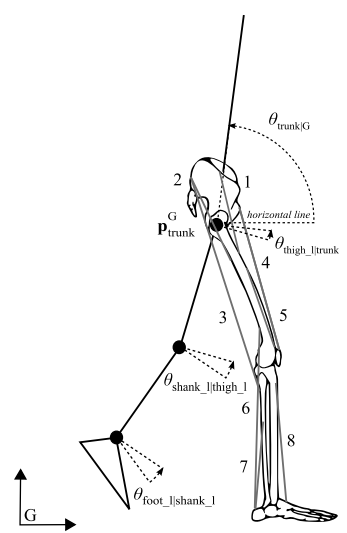

Fig. 1. Musculoskeletal model with seven rigid segments and 16 Hill-type muscles: 1 - iliopsoas, 2 - glutei, 3 - hamstrings, 4 - rectus femoris, 5 - vasti, 6 gastrocnemius, 7 - soleus, 8 - tibialis anterior. The model has nine kinematic

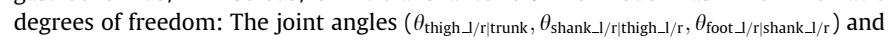
the global 2D position and sagittal orientation of the trunk $\left(\mathbf{p}_{\text {trunk }}^{G}, \theta_{\text {trunk } \mid G}\right)$.

as trunk, and three segments for each lower extremity (see Fig. 1). The segment masses, lengths, center of mass locations, and moments of inertia were estimated based on subject heights and body masses using the regression equations of Winter (2009). In total, the model had nine kinematic degrees of freedom summarized as generalized coordinates in vector $\mathbf{q}:=\left[\mathbf{p}_{\text {trunk }}^{G}, \theta_{\text {trunk } \mid G}, \theta_{\text {thigh_r } \mid \text { trunk }}, \theta_{\text {shank_r|thigh_r }}, \theta_{\text {foot_r|shank_r }}, \theta_{\text {thigh_l|trunk }}\right.$, $\left.\theta_{\text {shank_llthigh } \_l}, \theta_{\text {foot_llshank_l }}\right]^{T}$ comprising the $2 \mathrm{D}$ position of the trunk in global frame $G$, the sagittal orientation of the trunk relative to $G$, and the right and left leg joint angles in sagittal plane (see Fig. 1). We defined the time derivative of $\mathbf{q}$ as generalized velocities $\mathbf{v}$.

The model was actuated by 16 muscles, eight for each lower extremity as shown in Fig. 1. Each muscle was modeled as threeelement Hill-type model. The muscle contraction and activation dynamics are explained in van den Bogert et al. (2011). The state of each muscle was described by the length of the contractile element (CE) $L_{C E}$ and its activation $\alpha$. In addition, we defined two contact points at each foot (heel and toe) to model the foot-contact interaction. Each contact point introduced four state variables: the global position of the contact point $\left[c_{x}^{G}, c_{y}^{G}\right]^{T}$ and the global force exerted by the ground on the contact point $\left[F_{c_{x}}^{G}, F_{c_{y}}^{G}\right]^{T}$. A detailed formulation of contact dynamics can be found in supplementary material. Todorov (2010) altogether, the model's state was represented by the state vector:

$\mathbf{x}:=\left[\begin{array}{cl}\mathbf{q} & \text { nine generalized coordinates } \\ \mathbf{v} & \text { nine generalized velocities } \\ \mathbf{L}_{\mathbf{C E}} & 16 \text { contractile elements lengths } \\ \alpha & 16 \text { muscle activations } \\ {\left[c_{x, 1}, c_{y, 1}, F_{c_{x, 1}}, F_{c_{y, 1}}\right]^{T}} & \text { unknowns 1st contact point } \\ & \vdots \\ {\left[c_{x, 4}, c_{y, 4}, F_{c_{x, 4}}, F_{c_{y, 4}}\right]^{T}} & \text { unknowns last contact point }\end{array}\right]$.

The model was driven by the control vector $\mathbf{u}$ comprising the 16 neural excitations of the muscles. The system dynamics were implicitly formulated as function of $\mathbf{x}, \dot{\mathbf{x}}$, and $\mathbf{u}$ :

$\mathbf{f}(\mathbf{x}, \dot{\mathbf{x}}, \mathbf{u})=\mathbf{0}$. 
The function $\mathbf{f}()$ consisted of nine identities $\mathbf{v}-\dot{\mathbf{q}}=\mathbf{0}, 16$ muscle contraction and 16 activation dynamics equations (van den Bogert et al., 2011), 16 contact equations, and nine multi-body equations of motion (see supplementary material). The system dynamics $\mathbf{f}()$ were twice differentiable with respect to $\mathbf{x}, \dot{\mathbf{x}}$, and $\mathbf{u}$. This made it possible to use a gradient-based optimal control method in Section 2.3.

\subsection{Virtual sensor model}

We tracked accelerometer and gyroscope data with the planar musculoskeletal model in the optimal control simulation in Section 2.3. Therefore, we placed virtual sensors at the musculoskeletal model producing comparable accelerometer and gyroscope signals as would be measured by real sensors (see Fig. 2). We assumed that the IMUs were rigidly attached to the body segments $\mathrm{s} \in \mathcal{S}=\{$ trunk, thigh_r, shank_r, foot_r, thigh_l, shank_l, foot_l $\}$ at a known position $\mathbf{p}^{B_{s}}$ in the body-segment coordinate system $B_{s}$. Additionally, we ensured that the sensor axes were aligned with $B_{\mathrm{s}}$. The gyroscope signal in the sagittal-plane $\omega_{\mathrm{s}}$ was equal to the angular velocity of the corresponding body segment with respect to global frame $G$ and was calculated from the generalized velocities $\mathbf{v}$ :

$\omega_{\text {trunk }}:=\dot{\theta}_{\text {trunk } \mid G}$,

$\omega_{\text {thigh_r } / 1}:=\dot{\theta}_{\text {thigh_r } / l \mid G}=\dot{\theta}_{\text {thigh_r } / \mid \text { trunk }}+\dot{\theta}_{\text {trunk } \mid G}$,

$\omega_{\text {shank_r } / 1}:=\dot{\theta}_{\text {shank_r } /|| G}=\dot{\theta}_{\text {shank_r } /|| \text { thigh_r } / 1}+\dot{\theta}_{\text {thigh_r } /[\mid G}$,

$\omega_{\text {foot_r} / 1}:=\dot{\theta}_{\text {foot_r} / 1 \mid G}=\dot{\theta}_{\text {foot_r} / 1 \mid \text { shank_r } / 1}+\dot{\theta}_{\text {shank_r } / 1 \mid G}$.

An equation for the accelerometer signal $\mathbf{a}_{s}=\left[a_{x, s}, a_{y, s}\right]^{T}$ that would be measured at a body segment is given in van den Bogert et al. (1996). In 2D, the formula can be simplified to

$\mathbf{a}_{s}:=\mathbf{R}^{B_{\mathrm{s}} G}\left(\mathbf{a}_{G \mid B_{\mathrm{s}}}^{G}-\mathbf{g}\right)+\left[\begin{array}{cc}-\omega_{\mathrm{s}}^{2} & -\dot{\omega}_{\mathrm{s}} \\ \dot{\omega}_{\mathrm{s}} & -\omega_{\mathrm{s}}^{2}\end{array}\right] \mathbf{p}^{B_{\mathrm{s}}}$,

where $\mathbf{R}^{B_{S} G}$ is the transformation matrix from global frame to $B_{\mathrm{s}}, \mathbf{a}_{G \mid B_{\mathrm{s}}}^{G}$ is the acceleration of the segment origin relative to $\mathrm{G}$ (in G coordinates) and $\mathbf{g}=\left[0,-9.81 \mathrm{~m} / \mathrm{s}^{2}\right]^{T}$. We used Autolev 4.1 to generate a symbolic expression of Eq. (7) as a function of the kinematic states $\mathbf{q}, \mathbf{v}, \dot{\mathbf{v}}$ and sensor position $\mathbf{p}^{B_{s}}$. The time derivative $\dot{\mathbf{v}}$ was estimated using finite difference approximation.

\subsection{Optimal control problem}

The goal was to find the model states $\mathbf{x}(t)$ and controls $\mathbf{u}(t)$ during one gait cycle of duration $T$, such that the simulated acceleration and angular velocity corresponded to the measured signals

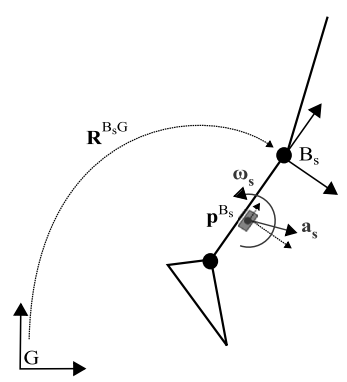

Fig. 2. Virtual sensors are placed at position $p^{B_{s}}$ in body-segment coordinate system $B_{\mathrm{s}}$. Angular velocity $\omega_{\mathrm{s}}$ and acceleration $a_{\mathrm{s}}$ are measured in $B_{\mathrm{s}}$ with respect to global frame $G$ with the transformation matrix $R^{B_{\mathrm{s}} G}$. without sensor noise. This was done by solving an optimal control problem following van den Bogert et al. (2011):

$$
\begin{array}{cl}
\underset{\mathbf{x}(t), \mathbf{u}(t)}{\operatorname{minimize}} & J(\mathbf{x}(t), \mathbf{u}(t)) \\
\text { subject to } & \mathbf{x}_{\mathrm{L}} \leqslant \mathbf{x} \leqslant \mathbf{x}_{\mathrm{U}} \\
& \mathbf{u}_{\mathrm{L}} \leqslant \mathbf{u} \leqslant \mathbf{u}_{\mathrm{U}} \\
& f(\mathbf{x}(t), \mathbf{x}(t), \mathbf{u}(t))=0 \\
& \mathbf{x}(0)+V T \mathbf{e}_{\mathrm{x}}-\mathbf{x}(T)=0,
\end{array}
$$

with the cost function $J(\mathbf{x}(t), \mathbf{u}(t))$ and the constraints on lower and upper bounds of $\mathbf{x}$ and $\mathbf{u}$ (see supplementary material), the dynamic equilibrium (Eq. (2)) and periodicity with a forward translation in direction $\mathbf{e}_{\mathrm{x}}$, where $\mathrm{T}$ was known $^{1}$ and the model's speed $V=\left(p_{x, \text { trunk }}^{G}(T)-p_{x, \text { trunk }}^{G}(0)\right) / T$ was unknown. The optimization problem was solved using direct collocation. Therefore, the state and control vectors were discretized at $N$ collocation nodes using backward Euler discretization. The cost function comprised a tracking term $J_{\text {track }}$, an effort term $J_{\text {effort }}$ and a regularization term $J_{\text {reg }}$ :

$J=J_{\text {track }}+J_{\text {effort }}+J_{\text {reg }}$.

In the tracking term, the squared difference between simulated signals (gyroscope: Eqs. (3)-(6), accelerometer: Eq. (7)) and corresponding measured signals was minimized. For this, we computed the mean $\mu$ of measured sensor signals over multiple strides (see Section 2.4.3). We normalized the differences to the measured standard deviation of multiple strides $\sigma$ to ensure that non-reproducible features such as noise and movement artifacts were not tracked:

$\left.\left.J_{\text {track }}=\frac{1}{|\mathcal{S}| N} \sum_{k=1}^{N} \sum_{s \in \mathcal{S}}\left(\frac{a_{\mathrm{x}, s k}-\mu_{a_{\mathrm{x}, s k}}}{\sigma_{a_{\mathrm{x}, s k}}}\right)^{2}+\frac{a_{\mathrm{y}, s k}-\mu_{a_{\mathrm{y}, s k}}}{\sigma_{a_{y, s k}}}\right)^{2}+\left(\frac{\omega_{s k}-\mu_{\omega_{s k}}}{\sigma_{\omega_{s k}}}\right)^{2}\right)$.

In this work, we tracked sagittal inertial signals of seven inertial sensors, one placed at each segment of the musculoskeletal model. However, it is also possible to reduce the number of sensors and, for example, track only signals of foot-mounted sensors. In the effort term, we minimized the squared excitations of all 16 muscles to resolve muscle redundancy and to prevent the model from tracking noisy measurements, which would require high muscular effort:

$J_{\text {effort }}=\frac{W_{\text {effort }}}{16 N V^{2}} \sum_{k=1}^{N} \sum_{m=1}^{16} u_{m k}^{2}$

where $W_{\text {effort }}$ is a weighting factor. Eq. (11) is similar to one used in previous work (van den Bogert et al., 2011), but we normalized the term with the squared speed of the model $V^{2}$. This was necessary to balance the tracking and effort terms when muscle activity increases over a wide range of walking and running speeds. We chose a quadratic normalization as previous work showed that muscle activity scales linearly with speed for walking and running (Belli et al., 1999; Neptune et al., 2008). The weighting was set empirically to $W_{\text {effort }}=300$ testing $W_{\text {effort }} \in\{1,100,200,300,400,500,1000\} . J_{\text {reg }}$ helped the convergence of the optimization and reduced the number of iterations by approximately $10 \%$. It was proportional to the integral of the sum of squares of the time derivatives of all state and control variables. We kept $J_{\text {reg }}$ small $\left(W_{\text {reg }}=1 e-5\right)$ so that the result of the optimization was not influenced. The large-scale nonlinear optimization problem of Eq. (8) was solved using IPOPT (Wächter and Biegler, 2006). As initial guess, we used a simulation of walking $\left(1.4 \mathrm{~m} \mathrm{~s}^{-1}\right)$ and running $\left(3.6 \mathrm{~m} \mathrm{~s}^{-1}\right)$ generated by tracking optical motion capture data from independent data sets.

\footnotetext{
${ }^{1}$ We computed $T$ from the average number of measurement points over multiple
} strides divided by the sampling frequency of the IMUs. 


\subsection{Evaluation study}

\subsubsection{Study design}

We conducted a study evaluating our inertial motion capturing (IMC) analysis against a gold-standard optical motion capturing (OMC) analysis. We used seven custom-built IMUs (Portabiles $\mathrm{GmbH}$, Erlangen, DE) (Blank et al., 2014) including tri-axial accelerometers $( \pm 16 \mathrm{~g})$ and gyroscopes $\left( \pm 2000 \mathrm{deg} \mathrm{s}^{-1}\right)$ sampled at $1000 \mathrm{~Hz}$. The OMC system consisted of 16 infrared cameras (Vicon MX, Oxford, UK) and one force plate (Kistler Instruments Corp, Winterhur, $\mathrm{CH}$ ), which were sampled at $200 \mathrm{~Hz}$ and $1000 \mathrm{~Hz}$, respectively. These systems were synchronized using a wireless flash trigger, which was received by every IMU and fed back to an analog channel of the OMC system (Kugler et al., 2012). A trigger signal was either sent manually or when the subjects crossed a light barrier which started the OMC recording.

In total, ten healthy male subjects volunteered for the study (age: $27.1 \pm 2.6$ years; height: $1.82 \pm 0.05 \mathrm{~m}$; weight: $76.9 \pm 8.6 \mathrm{~kg}$ ). Ethical approval for data collection was given and informed consent was obtained from all subjects before the study. We fixed seven IMUs on the subjects' bodies: at the lower back, right and left lateral thigh, lateral shank, and upper midfoot. The position of the IMUs was measured with respect to the segments' coordinate systems as required to compute simulated accelerometer signals in Eq. (7). In addition, we placed 32 infrared reflecting markers at anatomical landmarks.

At the beginning of the data acquisition, we asked the subjects to perform different movements in the sagittal-plane for estimating the orientation of the sensors with respect to the segments' coordinate systems (see Section 2.4.3). The trigger was activated before every movement, which were: standing, bending the torso forwards and backwards, lifting the straight leg up and down and flexing and extending the foot. In addition, a T-Pose, in which the subject stood still with arms stretched out to the sides, was recorded for a static calibration of the OMC system. The subjects were told to walk and run at six speed ranges: $0.9 \mathrm{~m} \mathrm{~s}^{-1}$ to $1.0 \mathrm{~m} \mathrm{~s}^{-1}$ (slow walking), $1.2 \mathrm{~m} \mathrm{~s}^{-1}$ to $1.4 \mathrm{~m} \mathrm{~s}^{-1}$ (normal walking), $1.8 \mathrm{~m} \mathrm{~s}^{-1}$ to $2 \mathrm{~m} \mathrm{~s}^{-1}$ (fast walking), $3.0 \mathrm{~m} \mathrm{~s}^{-1}$ to $3.3 \mathrm{~m} \mathrm{~s}^{-1}$ (slow running), $3.9 \mathrm{~m} \mathrm{~s}^{-1}$ to $4.1 \mathrm{~m} \mathrm{~s}^{-1}$ (normal running) and $4.7 \mathrm{~m} \mathrm{~s}^{-1}$ to $4.9 \mathrm{~m} \mathrm{~s}^{-1}$ (fast running). The subjects had to perform ten valid trials each, i.e., the speed range was controlled using the light barrier and the force plate had to be hit with the right foot.

\subsubsection{OMC data processing}

We filtered the marker and force plate data using a secondorder forward-backward low-pass Butterworth filter with a cutoff frequency of $10 \mathrm{~Hz}$ as suggested by Kristianslund et al. (2012). Afterwards, we downsampled the force plate data to $200 \mathrm{~Hz}$ and computed inverse kinematics and kinetics using the GaitAnalysisToolkit ${ }^{2}$ which implements the methods of Winter (2009). Then, computed joint angles, moments and GRFs were segmented into individual gait cycles and the step on the force plate was identified as first step after the trigger signal. We extracted the walking trials from right heel strike to right heel strike according to the minimum of the right heel marker. The running trials were cut from left toe-off to left toe-off using the method of Handsaker et al. (2016). We had to differentiate between walking and running to ensure that the gait cycle was completely captured by the infrared cameras and the stance phase on the force plate was completely included. However, this did not affect the analysis as the same time section was used for OMC and IMC analysis. The joint angles, joint moments and GRFs of the ten trials at each speed were linearly interpolated to the same length and averaged afterwards. Finally, we dec- imated the mean and variance to 100 sample points by linear interpolation for comparison to the IMC analysis.

\subsubsection{IMC data processing}

First, the axes of the IMU sensors were aligned with the segmental coordinate systems of the musculoskeletal model using the functional calibration movements (Ferraris et al., 1995). Afterwards, we extracted the strides that were recorded by the OMC system. The IMU data was cut using the detected start and end of the gait cycle (see Section 2.4.2) relative to the trigger signal. The ten trials of each speed were linearly interpolated to the same length and averaged afterwards. Finally, we decimated the mean and variance to $N=100$ sample points by linear interpolation for tracking the data with the musculoskeletal model (see Eq. (10)).

\subsubsection{Data analysis}

We computed the Pearson Correlation Coefficient $(\rho)$ between the estimated variables of the OMC and IMC analysis using all time points (from all subjects and speeds). Thus, no assumption on the distributions of the coefficients had to be made. In addition, we correlated gait cycles individually and took the mean using Fisher's z-transform for comparing our results to Karatsidis et al (2018). Here, we analyzed joint angles over the whole gait cycle and joint moments and GRFs over the stance phase. We categorized the correlation coefficients following Taylor (1990) into "weak" $(\rho \leqslant 0.35)$, "moderate" $(0.35<\rho<0.67)$, "strong" $(0.67<\rho<0.90)$, and "excellent" $(\rho \geqslant 0.90)$. In addition, we evaluated the absolute root-mean-square deviation (RMSD) of the joint angles, joint moments and GRFs in deg, in $\mathrm{BWBH} \%^{3}$ and $\mathrm{BW} \%$, respectively. The relative RMSD (rRMSD) was calculated as described in Ren et al. (2008). On the one hand, we used all samples and computed the RMSD applying ordinary least products (OLP) regression (Ludbrook, 1997). This removed the systematic error between the OMC and IMC method. On the other hand, we computed the RMSD and rRMSD without OLP regression for every single gait cycle individually and took the mean afterwards for comparison to Karatsidis et al. (2018).

\section{Results}

In total, 60 optimizations (ten subjects at six speeds) were solved with a mean CPU time of $50 \pm 26$ min on Intel Xeon processors E3-1240. In the optimization, we tracked the data of seven IMUs with a musculoskeletal model. The resulting simulated IMU signals had the same overall curve shape as the measured signals, whereas the oscillatory parts after heel strike were not tracked (see Fig. 3). The optimizations yielded natural walking and running simulations (see videos in the supplementary material). Kinematics and kinetics were compared to the OMC analysis. Fig. 4 shows the results for one subject. All individual results including muscle activations and forces can be found in the supplementary material.

Fig. 5 shows the correlation between OMC and IMC analysis based on all subjects and speeds. Gait kinematics yielded excellent correlations and gait kinetics $(\rho \geqslant 0.93)$ yielded strong to excellent correlation $(\rho \geqslant 0.90)$. In addition, Table 1 provides a comparison to Karatsidis et al. (2018) as described in Section 2.4.4.

\section{Discussion}

In this work, we presented a novel approach to estimate gait kinematics, kinetics and muscle forces by tracking IMU data with a trajectory optimization. This method addresses the two major 

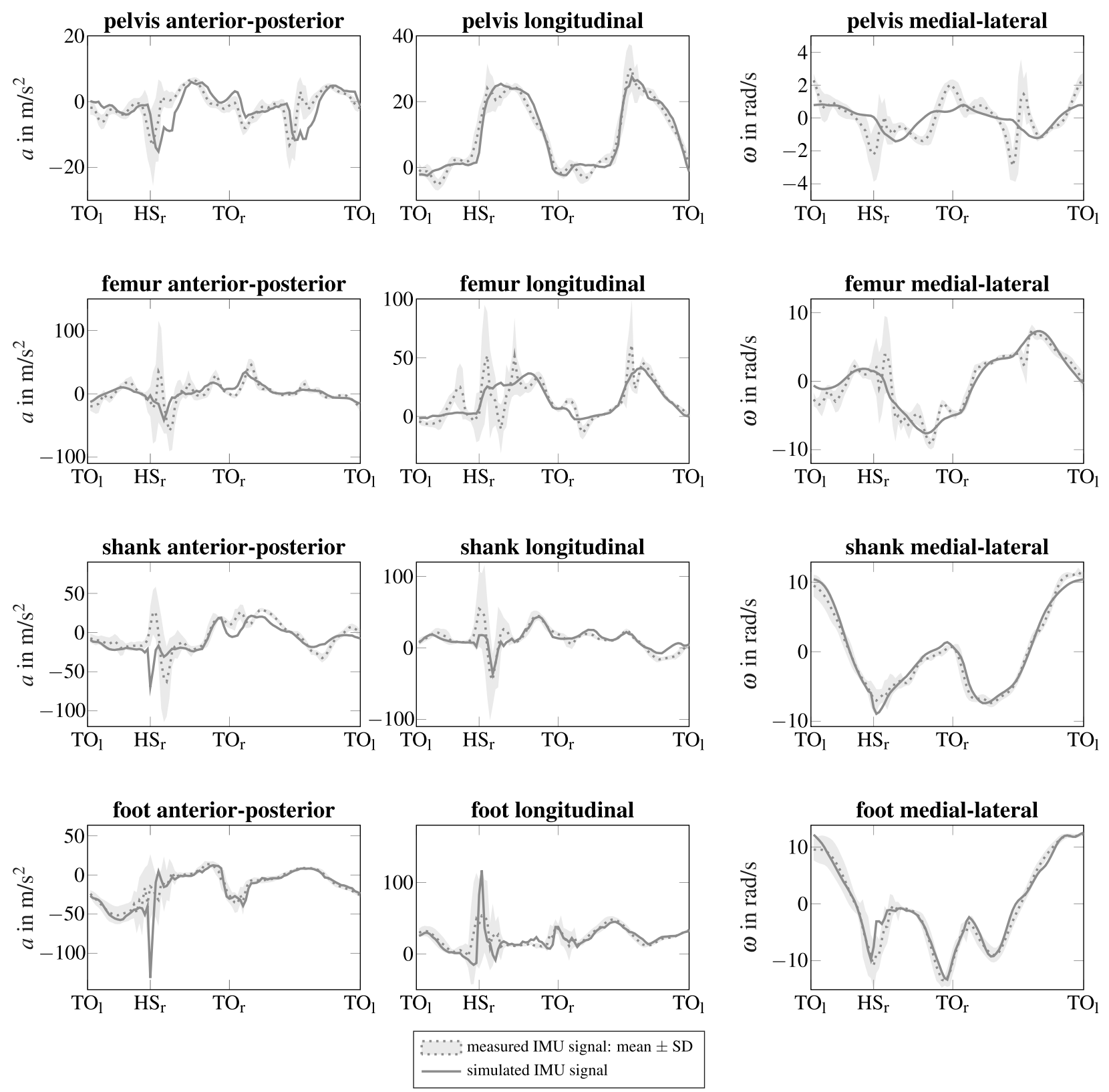

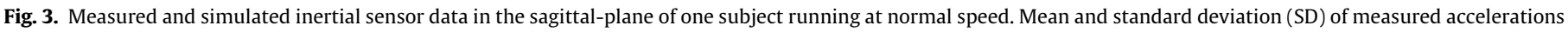

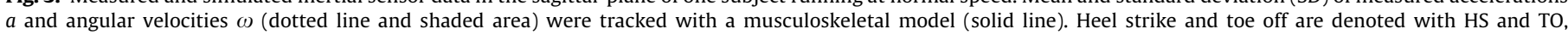
respectively.

challenges of IMC mentioned in the introduction (see Section 1), low-quality data and incomplete measurements.

We tracked raw sensor data without integrating the signals. Integration drift was avoided by using periodic boundary conditions and the physics of frictional contact to constrain translational movements. Moreover, Fig. 3 shows that movement artefacts in the IMU signals after heel strike were not tracked whereas features with a low standard deviation were tracked well (see for example the longitudinal shank acceleration). This is because we normalized the tracking term by data variance (see Eq. (10)), a model was used to eliminate solutions that are not dynamically consistent, and muscular effort was minimized (see Eq. (11)).

We estimated gait kinematics, kinetics, and muscle forces solely based on IMU data without ground reaction force data. Ground reaction forces (GRF) were indirectly estimated via a combination of a contact model, and the GRF-induced acceleration signals. The method would even allow fewer IMU sensors to be used. In preliminary work, we were able to reconstruct gait using only IMU data of foot-mounted sensors. However, results were worse and we would suggest to track additional variables such as normal joint angles and GRFs from the literature, to avoid unrealistic solutions which are still consistent with a subject's foot IMU data.

Results of the trajectory optimization were robust with respect to the weighting of the effort term in the cost function (Eq. (11)). For lower $W_{\text {effort }}$, joint angles improved slightly, whereas the resulting joint moments and GRFs got slightly worse. For higher $W_{\text {effort }}$, it was the other way round. Muscle activations were more noisy for lower $W_{\text {effort }}$, leading to noisy muscle forces. The normalization to squared speed allowed the same $W_{\text {effort }}$ to be used for all speeds, reducing the need for tuning of the cost function. In future 

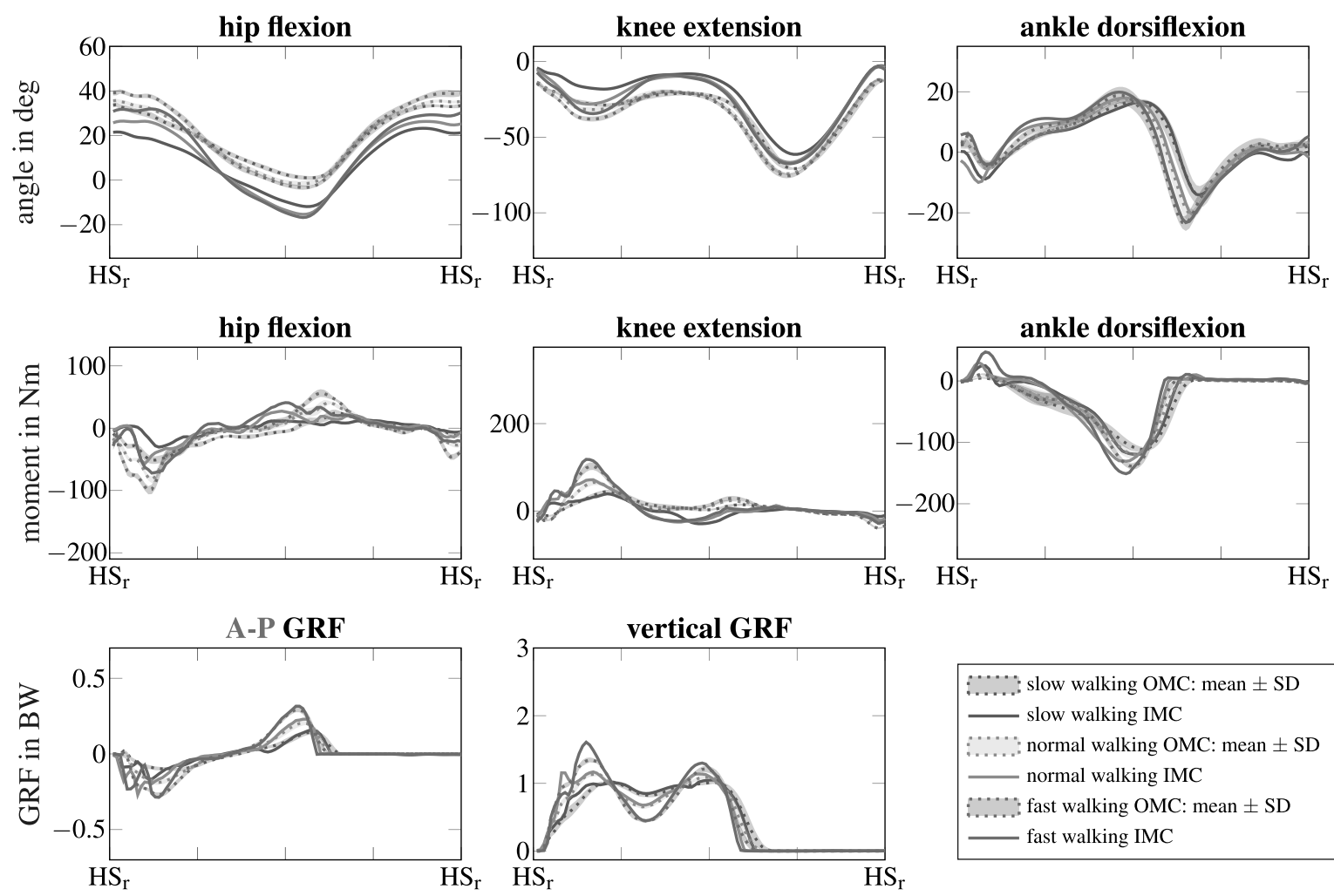

slow walking OMC: mean \pm SD

slow walking IMC

...... normal walking OMC: mean \pm SD normal walking IMC

$\ldots$ f....... fast walking OMC: mean \pm SD

- fast walking IMC

(a) Walking
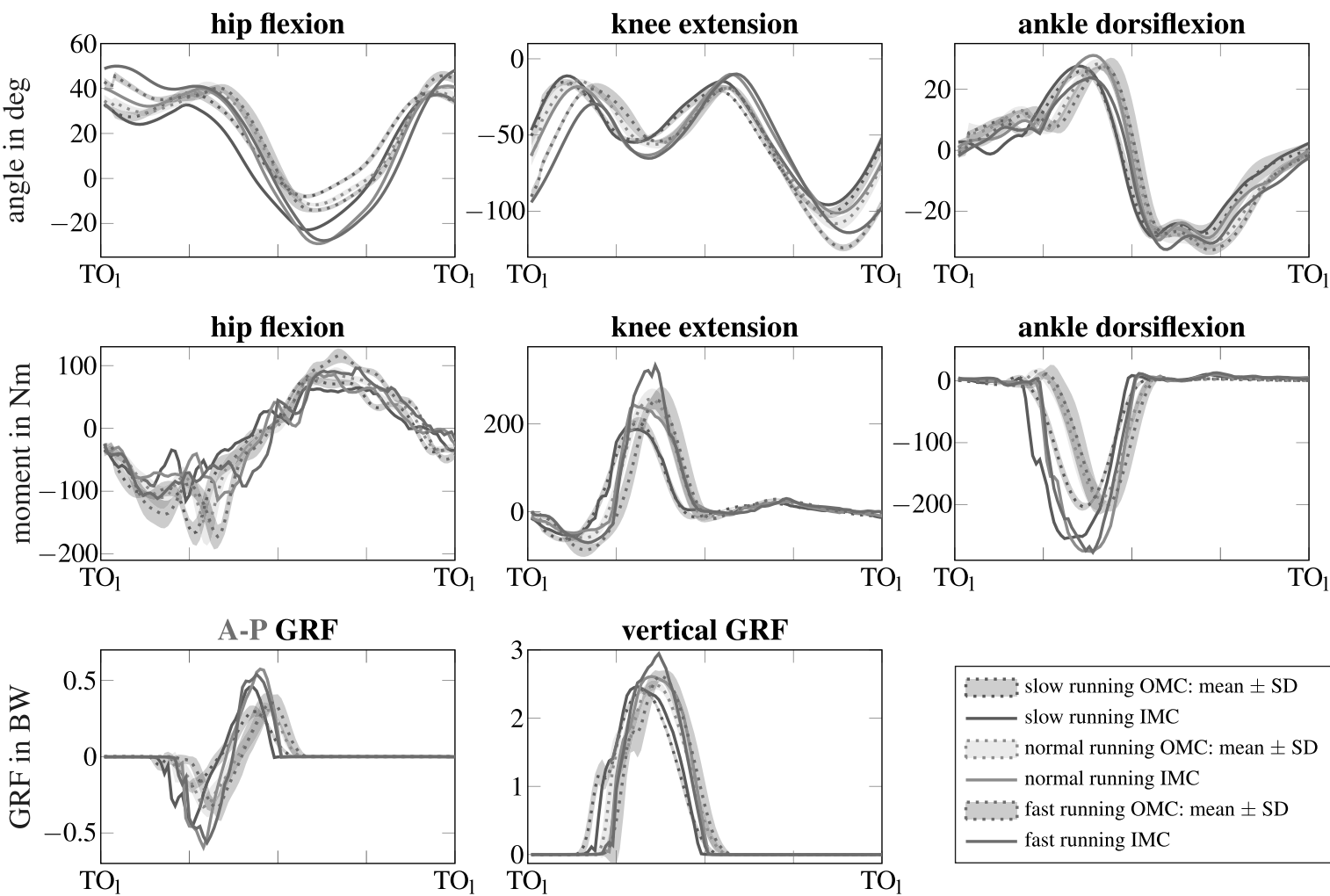

........ slow running OMC: mean $\pm \mathrm{SD}$

slow running IMC

$\ldots$ normal running OMC: mean \pm SD

normal running IMC

fast running OMC: mean \pm SD

- fast running IMC

(b) Running

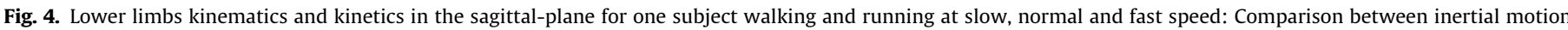

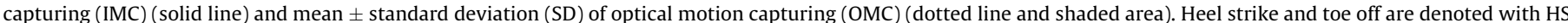
and $\mathrm{TO}$, respectively. 


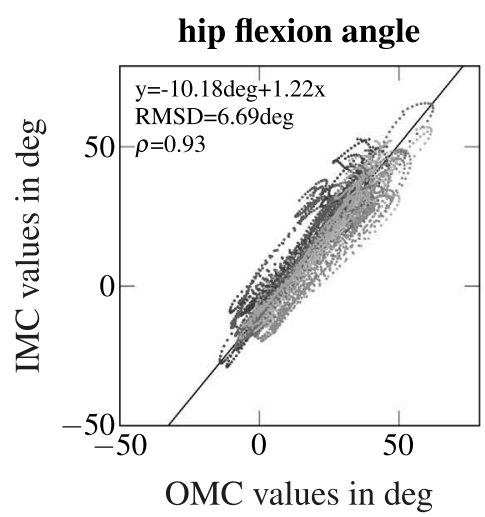

hip flexion moment

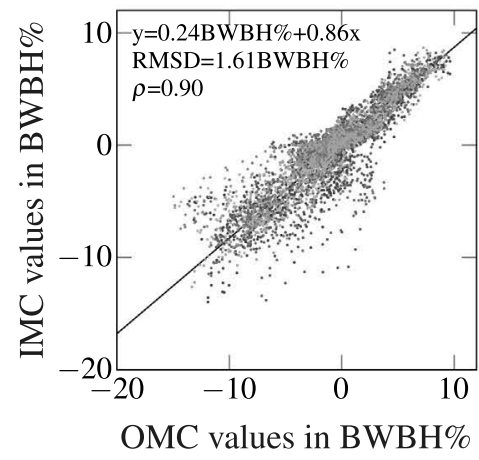

A-P GRF

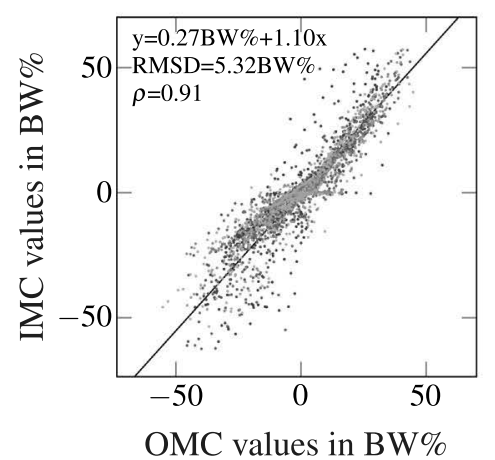

knee flexion angle

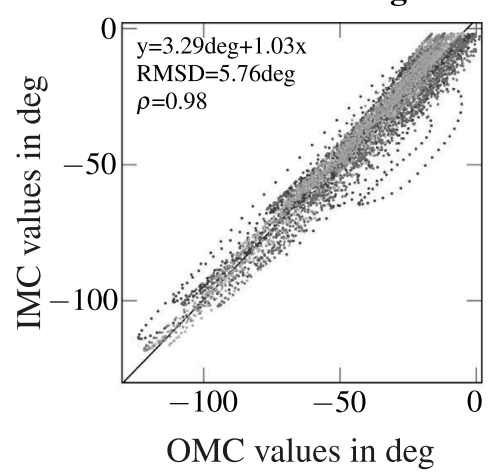

\section{knee extension moment}

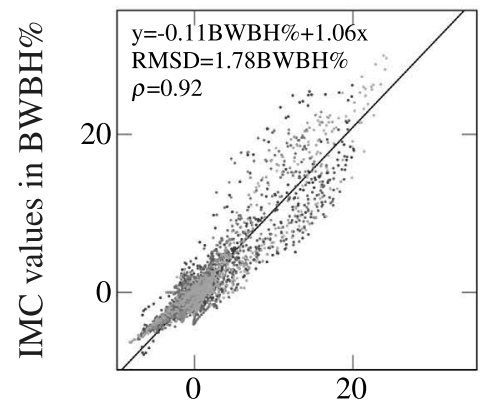

$\mathrm{OMC}$ values in $\mathrm{BWBH} \%$

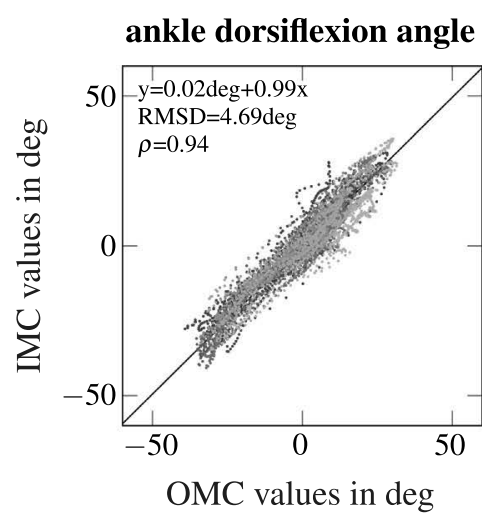

ankle dorsiflexion moment

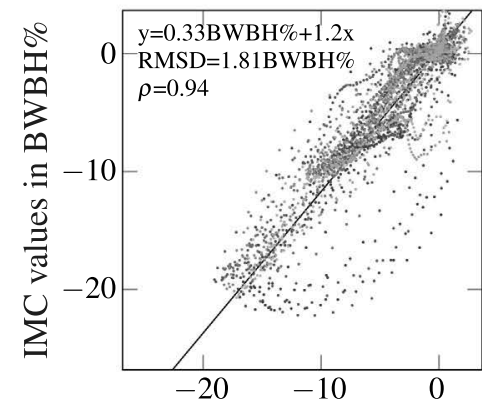

$\mathrm{OMC}$ values in $\mathrm{BWBH} \%$

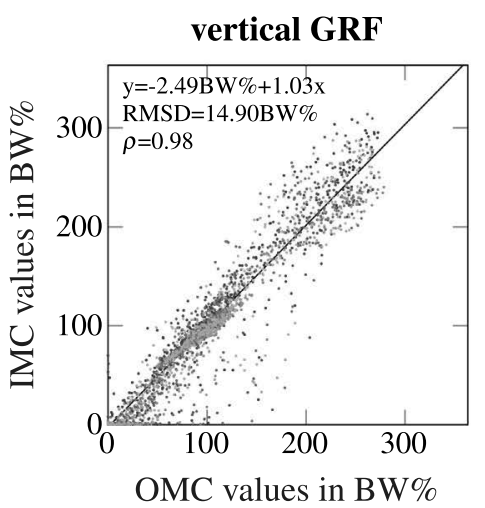

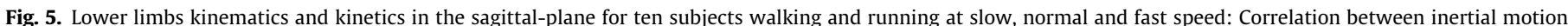

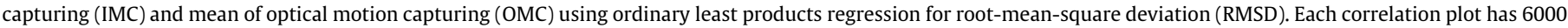
data points, from 10 subjects, 6 movements, and 100 collocation points. A-P GRF stands for anterior-posterior ground reaction force.

Table 1

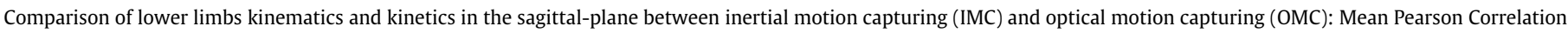

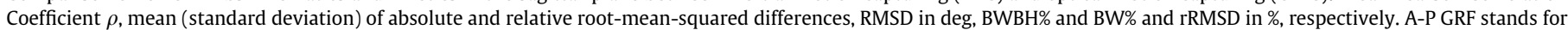
anterior-posterior ground reaction force.

\begin{tabular}{|c|c|c|c|c|c|c|c|c|c|c|c|c|c|c|c|}
\hline \multirow[b]{3}{*}{ Hip angle } & \multicolumn{5}{|c|}{ Walking } & \multicolumn{5}{|c|}{ Running } & \multicolumn{5}{|c|}{ Walking (Karatsidis et al., 2018) } \\
\hline & \multirow{2}{*}{$\frac{\rho}{1.00}$} & \multicolumn{2}{|c|}{ RMSD } & \multicolumn{2}{|c|}{ rRMSD } & \multirow{2}{*}{$\begin{array}{c}\rho \\
0.98\end{array}$} & \multicolumn{2}{|c|}{ RMSD } & \multicolumn{2}{|c|}{ rRMSD } & \multirow{2}{*}{$\begin{array}{c}\rho \\
0.99\end{array}$} & \multicolumn{2}{|c|}{ RMSD } & \multicolumn{2}{|c|}{ rRMSD } \\
\hline & & 8.2 & (3.3) & 21.9 & $(8.4)$ & & 8.7 & $(3.2)$ & 16.5 & $(5.5)$ & & 5.7 & $(2.1)$ & 12.7 & (5.3) \\
\hline Knee angle & 1.00 & 5.5 & $(2.8)$ & 8.8 & $(4.7)$ & 0.99 & 5.3 & $(3.0)$ & 6.1 & (3.8) & 0.99 & 4.4 & $(2.0)$ & 7.2 & (3.4) \\
\hline Ankle angle & 0.96 & 4.3 & (1.5) & 10.9 & $(3.4)$ & 0.98 & 4.6 & $(1.7)$ & 8.4 & (3.8) & 0.95 & 4.1 & $(1.3)$ & 14.0 & $(4.8)$ \\
\hline Hip moment & 0.76 & 1.5 & $(0.4)$ & 26.0 & $(9.8)$ & 0.85 & 3.2 & $(1.0)$ & 26.0 & (6.8) & 0.92 & 2.2 & $(0.6)$ & 19.4 & $(4.2)$ \\
\hline Knee moment & 0.81 & 1.5 & $(0.4)$ & 27.1 & $(9.2)$ & 0.94 & 3.4 & $(1.2)$ & 16.7 & (7.1) & 0.58 & 1.9 & $(0.5)$ & 29.8 & (7.6) \\
\hline Ankle moment & 0.95 & 1.6 & $(0.8)$ & 14.4 & $(6.8)$ & 0.96 & 3.2 & $(2.1)$ & 17.1 & $(10.8)$ & 0.93 & 1.6 & $(0.6)$ & 15.1 & (6.6) \\
\hline A-P GRF & 0.95 & 4.1 & $(1.2)$ & 9.7 & $(2.1)$ & 0.94 & 10.7 & $(3.9)$ & 13.5 & $(4.2)$ & 0.97 & 1.6 & $(0.6)$ & 17.5 & (6.8) \\
\hline Vertical GRF & 0.95 & 11.1 & (3.4) & 9.6 & $(2.5)$ & 0.94 & 32.0 & $(7.9)$ & 12.8 & (3.6) & 0.91 & 9.3 & $(3.0)$ & 7.7 & $(2.1)$ \\
\hline
\end{tabular}


work, other effort measures could be used, such as metabolic cost (Koelewijn et al., 2018). Another possibility would be to learn the objective function from data (Clever et al., 2016).

The musculoskeletal model had several limitations that are relevant to the results of the study. The two-dimensional model limited the analysis to sagittal plane variables and muscles, but also may have affected the ability of the model to track IMU data. For instance, the model does not account for the effect of frontal plane rotation of the pelvis on the vertical accelerometer signal of the lower back IMU sensor. Our method can be used in a full 3D model to overcome these limitations, albeit at the expense of longer computation time to solve the trajectory optimization problem. The musculoskeletal model had a rigid foot, which seems to have affected the running results. During running, the ankle plantarflexion moment rose too quickly, compared to the OMC results. We believe that the IMU on the upper midfoot picked up an anterior pitch rotation, which the contact model then interpreted as an anterior shift in the center of pressure. A model with a deformable foot may have prevented this error. Similarly, the pelvis and upper body were represented by one rigid segment. The lower back IMU was placed on the sacrum and likely not representative of upper body dynamics, resulting in poor estimates of hip angle and moment. This error could be reduced by tracking additional upper body IMU sensors, possibly even without additional kinematic degrees of freedom. Another fundamental limitation is that the trajectory optimization approach uses a minimal effort criterion to distribute the effort among the muscles that actuate the joints. Unequal load distribution, and "unnecessary" antagonistic cocontraction will likely be missed. Integrating EMG sensing with IMU sensing will be necessary to resolve this issue.

Fig. 5 shows how the IMC results correlate to conventional OMC results, across subjects, across walking/running conditions, and across time points in the gait cycle. The overall correlation coefficients are all above 0.9 , indicating that $80 \%$ of the true variance is predicted by the IMC analysis. Some gait cycles from the running tests seem to be outliers in the correlation diagram, and we suspect that these are local minima of their respective trajectory optimization problems. This could be alleviated by solving with multiple initial guesses, rather than one. The RMS differences between IMC and OMC are small enough for clinical applications where changes of 5-10 degrees in joint angle are clinically relevant (Khouri and Desailly, 2013). We suspect, however, that the correlations and RMS errors will be better for typical clinical study designs, where we are interested in differences between subjects (e.g. patient vs. normal) and even more so to determine withinsubject changes such as treatment effects.

Compared to Karatsidis et al. (2018), we achieved similar performance (see Table 1) while reducing the number of software tools and processing steps. Our approach does not require a separate kinematic and kinetic analysis, combining the two into a single dynamic trajectory optimization. This has the advantage that no error propagation occurs and a dynamically consistent simulation is obtained. Thus, highly dynamic movements like running can be analyzed.

Similar to classical inverse dynamics, the IMU tracking approach has the limitation that individual muscle forces are resolved through an optimality criterion. In the present study, this is the effort term in the optimization objective. In pathological conditions where muscle force distribution is non-optimal (such as neurological disorders), muscle forces will be underestimated. In addition, any approach without force plate data will not uniquely resolve the load distribution between the two feet in a double support phase. This might affect clinical applications, such as stroke, where limb loads are not distributed optimally. Our validation study used able-bodied participants, so clinical validity is still an open question. In future work, the trajectory optimization approach can include EMG data in the tracking term of the optimization objective, in order to obtain solutions that are consistent with measured motion and EMG.

Trajectory optimization is fundamentally not a real-time analysis, because the optimization cannot start until data from an entire movement cycle has been collected. Higher computing power or larger convergence tolerances of the IPOPT would reduce computing time, and results may be available in near real time. On the other hand, three-dimensional models will eventually be needed which would likely increase the computation time dramatically.

In conclusion, gait kinematics and kinetics could be estimated for a wide range of speeds and subjects. We tracked inertial sensor data with a musculoskeletal model by solving an optimal control problem and obtained a dynamically consistent simulation. The method suppresses sensor artifacts and is drift-free. It provides a comprehensive gait analysis solely based on IMU data with a single software tool.

\section{Declaration of Competing Interest}

None of the authors had any financial or personal conflict of interest with regard to this work.

\section{Acknowledgement}

The authors thank Iris Kellermann for preliminary work and Heiko Schlarb for supporting the data acquisition. This research was supported by the Bavarian Ministry of Economic Affairs, Regional Development and Energy within the Embedded Systems Initiative. Bjoern Eskofier gratefully acknowledges the support of the German Research Foundation (DFG) within the framework of the Heisenberg professorship program (Grant No. ES 434/8-1).

\section{Appendix A. Supplementary material}

Supplementary data associated with this article can be found, in the online version, at https://doi.org/10.1016/j.jbiomech.2019.07. 022 .

\section{References}

Ancillao, A., Tedesco, S., Barton, J., O’Flynn, B., 2018. Indirect measurement of ground reaction forces and moments by means of wearable inertial sensors: systematic review. Sensors 18 (8), 2564.

Belli, A., Kyrolainen, H., Komi, P.V., 1999. Changes in muscle activity patterns and kinetics. Strength Cond. 13 (4), 400-406.

Blank, P., Kugler, P., Eskofier, B.M., 2014. A wearable sensor system for sports and fitness applications. In: Book of Abstracts of the 20th Annual Congress of the European College of Sport Science (ECSS). Amsterdam, The Netherlands, p. 703 available online: https://www5.informatik.uni-erlangen.de/Forschung/ Publikationen/2014/Blank14-AWS.pdf.

Clever, D., Schemschat, R.M., Felis, M.L., Mombaur, K., 2016. Inverse optimal control based identification of optimality criteria in whole-body human walking on level ground. In: Proceedings of the 6th IEEE International Conference on Biomedical Robotics and Biomechatronics (BioRob). University Town Singapore, pp. 1192-1199.

Ferraris, F., Grimaldi, U., Parvis, M., 1995. Procedure for effortless in-field calibration of three-axis rate gyros and accelerometers. Sensors Mater. 7, 311.

Handsaker, J.C., Forrester, S.E., Folland, J.P., Black, M.I., Allen, S.J., 2016. A kinematic algorithm to identify gait events during running at different speeds and with different footstrike types. J. Biomech. 49 (16), 4128-4133.

Hannink, J., Kautz, T., Pasluosta, C.F., Gaßmann, K.-G., Klucken, J., Eskofier, B.M. 2016. Sensor-based gait parameter extraction with deep convolutional neural networks. IEEE J. Biomed. Health Informat., 1-8

Heinrich, D., van den Bogert, A.J., Nachbauer, W., 2014. Relationship between jump landing kinematics and peak ACL force during a jump in downhill skiing: a simulation study. Scand. J. Med. Sci. Sports 24 (3), 180-187.

Karatsidis, A., Jung, M., Schepers, H.M., Bellusci, G., de Zee, M., Veltink, P.H. Andersen, M.S., 2018. Predicting kinetics using musculoskeletal modeling and inertial motion capture. arXiv preprint arXiv: 1801.01668

Khouri, N., Desailly, E., 2013. Rectus femoris transfer in multilevel surgery: technical details and gait outcome assessment in cerebral palsy patients. Orthopaed. Traumatol. Surg. Res. 99 (3), 333-340. 
Koelewijn, A.D., Dorschky, E., van den Bogert, A.J., 2018. A metabolic energy expenditure model with a continuous first derivative and its application to predictive simulations of gait. Comput. Methods Biomech. Biomed. Eng. 21 (8), 521-531.

Kok, M., Hol, J.D., Schön, T.B., 2014. An optimization-based approach to human body motion capture using inertial sensors. IFAC Proc. Vol. 47 (3), 79-85.

Koning, B.H.W., van der Krogt, M.M., Baten, C.T.M., Koopman, B.F.J.M., 2013. Driving a musculoskeletal model with inertial and magnetic measurement units. Comput. Methods Biomech. Biomed. Eng. 18 (9), 1003-1013.

Kristianslund, E., Krosshaug, T., van den Bogert, A.J., 2012. Effect of low pass filtering on joint moments from inverse dynamics: implications for injury prevention. J. Biomech. 45 (4), 666-671.

Kugler, P., Schlarb, H., Blinn, J., Picard, A., Eskofier, B., 2012. A wireless trigger for synchronization of wearable sensors to external systems during recording of human gait. In: Proceedings of the 34th Annual International Conference of the IEEE Engineering in Medicine and Biology Society (EMBC). San Diego, California, USA, pp. 4537-4540.

Liedtke, C., Fokkenrood, S.A., Menger, J.T., van der Kooij, H., Veltink, P.H., 2007. Evaluation of instrumented shoes for ambulatory assessment of ground reaction forces. Gait Posture 26 (1), 39-47.

Liu, T., Inoue, Y., Shibata, K., 2010. A wearable force plate system for the continuous measurement of triaxial ground reaction force in biomechanical applications. Meas. Sci. Technol. 21 (8).

Ludbrook, J., 1997. Special article comparing methods of measurement. Clin. Exp. Pharmacol. Physiol. 24 (2), 193-203.

Neptune, R.R., Sasaki, K., Kautz, S.A., 2008. The effect of walking speed on muscle function and mechanical energetics. Gait Posture 28 (1), 135-143.

Prilutsky, B.I., Zatsiorsky, V.M., 2002. Optimization-based models of muscle coordination. Exerc. Sport Sci. Rev. 30 (1), 32-38.

Ren, L., Jones, R.K., Howard, D., 2008. Whole body inverse dynamics over a complete gait cycle based only on measured kinematics. J. Biomech. 41 (12), 2750-2759.
Roetenberg, D., Luinge, H., Slycke, P., 2009. Xsens mvn: full 6dof human motion tracking using miniature inertial sensors. Xsens Motion Technologies BV, Tech. Rep 1.

Roetenberg, D., Luinge, H.J., Baten, C.T., Veltink, P.H., 2005. Compensation of magnetic disturbances improves inertial and magnetic sensing of human body segment orientation. IEEE Trans. Neural Syst. Rehabil. Eng. 13 (3), 395-405.

Sabatini, A.M., Ligorio, G., Mannini, A., 2015. Fourier-based integration of quasiperiodic gait accelerations for drift-free displacement estimation using inertial sensors. Biomed. Eng. Online 14 (1), 106.

Schepers, H., van Asseldonk, E., Buurke, J., Veltink, P., 2009. Ambulatory estimation of center of mass displacement during walking. IEEE Trans. Biomed. Eng. 56 (4), 1189-1195.

Taylor, R., 1990. Interpretation of the correlation coefficient: a basic review. J. Diagn. Med. Sonog. 6 (1), 35-39.

Todorov, E., 2010. Implicit nonlinear complementarity: A new approach to contact dynamics. In: Proceedings of the IEEE International Conference on Robotics and Automation (ICRA), Anchorage, Alaska, USA, pp. 2322-2329.

van den Bogert, A.J., Blana, D., Heinrich, D., 2011. Implicit methods for efficient musculoskeletal simulation and optimal control. Procedia IUTAM 2, 297-316.

van den Bogert, A.J., Read, L., Nigg, B.M., 1996. A method for inverse dynamic analysis using accelerometry. J. Biomech. 29 (5), 667-671.

Wächter, A., Biegler, L.T., 2006. On the implementation of an interior-point filter line-search algorithm for large-scale nonlinear programming. Math. Program. 106 (1), 25-57.

Winter, D.A., 2009. Biomechanics and Motor Control of Human Movement. John Wiley \& Sons.

Wouda, F.J., Giuberti, M., Bellusci, G., Maartens, E., Reenalda, J., van Beijnum, B.J.F., Veltink, P.H., 2018. Estimation of vertical ground reaction forces and sagittal knee kinematics during running using three inertial sensors. Front. Physiol. 9, $1-14$.

Post-print standardized by MSL Academic Endeavors, the imprint of the Michael Schwartz Library at Cleveland State University, 2019 